\title{
THE ABUNDANCE OF HELIUM IN STELLAR INTERIORS*
}

\author{
JOHN FAULKNER \\ Lick Observatory, Board of Studies in Astronomy and Astrophysics, \\ University of California, Santa Cruz, Calif., U.S.A.
}

\section{Introduction}

From the titles of the other contributions to this morning's session, one deduces that the hard facts of theory are to be sandwiched between the loose speculations of direct observation. I shall therefore feel free to discuss theoretical implications from stellar structure unencumbered by what are, after all, but peripheral considerations.

I should, however, begin with an apology. It is impossible to do even minimal justice to the vast number of investigations which have contributed to our present understanding (or lack of it). The choice of topics has been dictated in part by history, in part by current relevance, but mainly by personal whim. While much of the discussion centres, for obvious reasons, on Population II work, one or two problems associated with Population I will also be considered. Reference may be made to the exhaustive review article of Iben (1967) and also to the Warner Prize Lecture by Demarque (1968) for background and a more complete discussion of some of the points which will be touched upon here.

I shall assume today that the 'normal' helium content of stars is of the order of $25 \pm 5 \%$ by mass. This leaves me free to pay particular attention to systems which do not appear to adhere to this generalization, systems for which abnormally high or low helium contents have been claimed. The main point of my talk is that there is no really convincing, concrete evidence from stellar interiors alone for helium contents far removed from normal. During the course of the past decade, this has come to be largely accepted. It is in a way sad to see the radicalism of one's youth become the orthodoxy of middle age.

\section{The Main-Sequence HR and ML Diagrams}

The earliest approach of stellar model investigators to the helium problem lay in computing main-sequence (homogeneous) stellar models and comparing the positions of computed sequences with those observed. Putting aside for one moment the observational difficulties, the positions of the sequences are, in zeroth order, sensitive to both helium and metal contents. If an assumption about the metal content is made, comparison in either diagram should give the helium content. In fact, as the sensitivities are different in the two diagrams no assumption about the metal content is actually necessary - one simply looks for the self-consistent solution to both problems (Faulkner, 1964; Bodenheimer, 1965; Iben, 1967).

* Contributions from the Lick Observatory, No. 333. 
In practice the comparisons are rendered uncertain by both theoretical and observational difficulties. For late-type stars the theoretical model radii (and hence effective temperatures) are uncertain because of the lack of a definitive treatment of the outer convection zone. Observationally, errors in distance moduli affect both diagrams, while any errors in deduced masses can strongly affect the ML diagram where theory makes its most unambiguous predictions. In saying this one assumes, perhaps unjustifiably, that the bolometric corrections and colour-temperature relations are known to sufficient accuracy, and furthermore that evolutionary effects are either insignificant or correctly accounted for.

Nevertheless, in spite of these difficulties, there are Population I systems for which the comparisons may fairly be made. Almost immediately, the theory of stellar structure is faced with enormous difficulties if the observational data are correct. I refer, of course, to the problem of the Hyades.

Eggen, in a series of investigations during the last decade (Eggen, 1962, 1963, 1969) claims to observe two distinct mass-luminosity relations for Population I stars. Those having a solar-type ultra-violet excess with respect to the Hyades occupy the 'SunSirius' relation, while those with essentially no ultra-violet excess occupy the 'HyadesPraesepe' or 'Hyades group' relation. For stars of supposedly solar mass, the latter relation is brighter by 1.5 or more. This has lead to suggestions that the Hyades may be richer in helium than the Sun by factors ranging from 1.5 to 2 (Eggen, 1962; Faulkner, 1964; Bodenheimer, 1965). However, the simultaneous requirement for consistency in the HR diagram cannot be met, and the model Hyades stars are underluminous by 0.7 to 1.0 or more. It has been suggested that some of the difficulties may be removed, or at least eased, by increasing the distance modulus of the Hyades by up to 0.4 (Hodge and Wallerstein, 1966; Faulkner, 1964, 1967; Iben, 1967). However, a bodily shift of the Hyades as a whole may be unnecessary. It still appears that the binaries used to establish the ML relation are slightly underluminous, and closer to us than the average Hyades cluster member. Since masses are given by

$$
M_{1}+M_{2}=\frac{a^{3}}{\pi^{3} P^{2}},
$$

one may appeal to systematic errors in both $\pi$ and $a$ (assuming $P$ is well-known) to move the observations along error vectors (e.g. those labelled $\pi^{3}$ or $a^{3}$ in Figure 1). Eggen (1969) claims that the estimated probable errors preclude shifts of the required magnitude. However, his own data for the Sirius group changed dramatically and systematically between 1962 and 1963 when the group relation became fainter by $\sim 0$.75. Earlier moving group parallaxes had been overestimated by $\Delta \pi \sim 0.003(\mathrm{~Pa}-$ rallaxes were in the range $0^{\prime \prime} 040 \lesssim \pi \lesssim 0^{\prime \prime} .060$ ). Thus each individual star was more luminous than had been thought (a glance at Figure 1 shows that changing $\pi$ such that an individual star becomes brighter makes the group relation become fainter a statement which is not the paradox it would at first appear to be).

One should realise that errors in both $\pi$ and $a$ are greatly magnified in the ML diagram. In Figure 1 we show the Sun with a representative Hyades point, $H$, and 


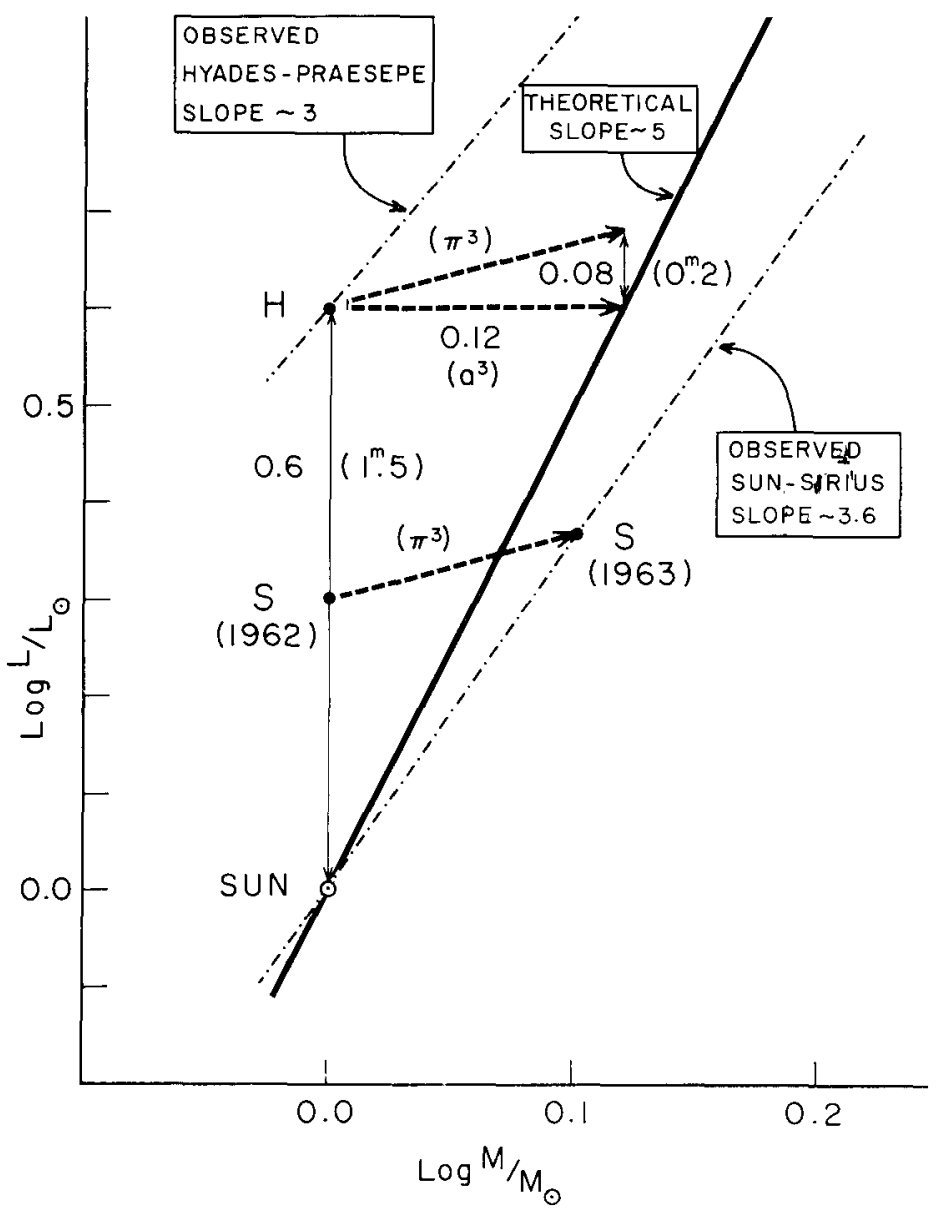

Fig. 1. A schematic mass-luminosity diagram (see text).

Sirius points $S . H$ is $1^{\mathrm{m}} .5$ brighter than the Sun, through which we draw the theoretical slope of about 5 for stars of $\sim 1 M_{\odot}$. It should be noted that the lower slope ( $\lesssim 3.6$ ) obtained by Eggen for the Sirius group is weighted with somewhat more massive stars. Theoretically, the slope varies from $\sim 5$ at $\sim 1 M_{\odot}$ through $\sim 4$ at $\sim 2.5 M_{\odot}$ and on to still lower values. In the range $1 M_{\odot}-2.5 M_{\odot}$ there seems in fact to be excellent agreement with the recent results obtained by Popper et al. (1970) from the admittedly inhomogeneous sample provided by the seven best 'unevolved' main-sequence eclipsing binaries. The latter authors incidentally find $N_{\mathrm{He}} / N_{\mathrm{H}} \sim 0.12 \pm$ \pm 0.02 , i.e. helium contents slightly in excess of $\sim 30 \%$ by mass.

Taking then the theoretical slope through the solar point rather than an empirically determined mean lower value, it turns out that the issue of the helium richness theoretically rests on an error of $\sim 0.04$ in $\log (a / \pi)$, where the relative apportionment of the $10 \%$ error is not very critical in view of the shallow slope of $\pi$-induced errors $\left(\frac{2}{3}\right)$ in comparison with the theoretical slope of 5 . 
The value of $\pi$ appears to be well settled (Wayman et al. 1965) although Wayman (1967) in showing how unlikely the Hodge and Wallerstein shift of 0.4 may be, gives figures which do not appear to rule out a possible $0 \mathrm{~m} 2$ shift. Eggen (1969) has objected strongly to suggestions by, among others, Alexander (1968) that the double star observations of his selected observers could possibly be in error by the required amounts.

While on this topic, I should point out the further difficulties encountered theoretically when trying to understand the slope $(\$ 3)$ attributed to the Hyades solarmass stars by Eggen. The slope may of course be spurious since it comes about by linking the 'reliable' Hyades binaries with members of the Hyades moving group and Praesepe, not necessarily the homogeneous sample Eggen supposes. (One notes in passing that the reliable binaries have been reduced in number from 6 in 1962 to 3 in 1969, and looks forward with interest to 1976.) Alexander (1968) on the other hand, choosing what he considered 'better' orbits (hotly disputed by Eggen) obtained results for the Hyades binaries alone from which he denies the possibility of deducing a slope in view of the small range covered. Since the value one might deduce from his data would be only of order $\sim 1-1.5$, it would certainly be extremely unpalatable.

To illustrate the theoretical abhorrence for such low slopes, consider the ML relation produced by homologous stars built with physical laws of the form

$$
\begin{aligned}
\kappa & =\kappa_{0} \varrho^{\lambda-1} T^{3-\vartheta} \\
\varepsilon & =\varepsilon_{0} \varrho T^{\eta}
\end{aligned}
$$

which should be good enough for small mass ranges.

Then

$$
L \propto M^{n}
$$

where

$$
n=\frac{2 \lambda \eta+3 \lambda+3+v+\eta}{3+3 \lambda+\eta-v} .
$$

If Kramer's opacity holds, $\kappa=\kappa_{0} \varrho T^{-3.5}$ i.e. $\lambda=2, v=6.5$. Then $n=(10 \eta+31) /(2 \eta+5)$. For the $p p$-chain with $\eta \simeq 4, n \simeq 5.5$. In any case, for all $\eta>0,5<n<6.2$. To gain further illumination, let us suppose, with Eggen, that the observations indeed give us $n=3$. Let us also suppose that the energy generation gives us $\eta=4$ or 6 (a realistic range for the $p p$-chain). We then ask what opacity laws could give us Eggen's slope. The results are as follows:

\begin{tabular}{ll} 
Opacity laws consistent with $L \propto M$ \\
$n=4$ & $n=6$ \\
$\quad \vdots$ & $\vdots$ \\
$\kappa_{0} \varrho^{-1} T^{-0.5}$ & $\kappa_{0} \varrho^{-1} T^{-1.5}$ \\
$\kappa_{0}$ & $\kappa_{0}$ \\
$\kappa_{0} \varrho T^{0.5}$ & $\kappa_{0} \varrho T^{1.5}$ \\
$\kappa_{0} \varrho^{2} T$ & $\kappa_{0} \varrho^{2} T^{3}$ \\
\multicolumn{1}{c}{$\vdots$} & $\vdots$
\end{tabular}


We see that electron-scattering would work - but it is of course thought negligible compared to the bound-free and free-free contributions for models in this range. The other entries seem to require unacceptable temperature dependencies. Entries preceding $\kappa_{0}$ have negative power dependencies for both $\varrho$ and $T$. This density dependence invites one to think of conductive opacities dominating, but the temperature dependence goes the wrong way (Hubbard and Lampe, 1969). In any case, while some judicious combination of conductive and radiative opacities might mimic the above dependencies, it seems most unlikely to occur in the required mass range. Thus no simple solution to the problem posed by Eggen's slope of 3 seems to exist. Either stellar structure (via the opacities), or the observations (via orbits or parallaxes) will have to prove flexible in the future.

Meanwhile, let me emphasize as strongly as possible that until this problem of the low slope is cleared up, it is a meaningless and futile exercise to attempt to deduce the helium content of the Hyades - unless, that is, one is prepared to believe in the strong function of mass which the comparisons would at present demand!

Before leaving the main sequence in the HR diagram, I should mention briefly attempts to determine the helium content of subdwarfs. Eggen and Sandage (1962) claimed that corrections for line blanketing placed the subdwarfs of known parallax on the same line as the Hyades in the $M_{\text {bol }}, \log T_{\mathrm{e}}$ plane. On the basis of crude homology expressions, this seemed to require low helium content to compensate for the low metal abundance. Doubts on the complete correction, particularly for extreme subdwarfs, were expressed (Faulkner, 1967), and an examination of the residual effect with improved quasi-homology representations suggested that subdwarf helium contents differed little from the solar value. This conclusion was greatly strengthened by the work of Strom and Strom (1967) who compared spectrum scans in the range $5000 \AA-7500 \AA$ with the predictions of model atmospheres to calibrate the observed ultra-violet excesses and colour-temperature relations. They concluded that the subdwarfs were indeed underluminous, and that the choice of $Y \sim 0$ was essentially ruled out. Cayrel (1968) subsequently used the more suitable late-type colour indices (viz. GRI, vby, VRIJK) to show a clear segregation, with the main sequence of extreme subdwarfs underluminous by $\sim 0.7 \pm 0$. 3 . Comparison with model calculations suggested once again that helium was 'normal'.

One final main sequence puzzle is that associated with the helium-weak B stars in Orion (Sargent and Strittmatter, 1966). These sharp-lined stars lie on the lower edge, or below, the normal main sequence defined by other stars in their neighbourhood, a circumstance which from stellar interiors would require them to be non-rotating stars of possibly higher than normal helium. The low abundance interpretation of the spectra was questioned by some since non-LTE processes were thought to be responsible for the singlet-triplet $\mathrm{He}$ I anomaly found in both these and some galactic halo stars (Sargent and Searle, 1968). However, Norris (1970a) has explained the anomaly as an LTE saturation effect which still requires that the surface value of $N_{\mathrm{He}} / N_{\mathrm{H}}$ be lower than normal by factors of $\sim 2-15$ possibly (Norris, 1970b). For these Population I stars, the weakness seems to occur provided helium is not all ionized, 
agreeing with Michaud's theory (Michaud, 1970) of diffusion and gravitational settling which accounts for many of the Ap spectral peculiarities.

While this is an attractive explanation, it may not be the whole story however. The Population II B subdwarfs which also look weak are apparently too hot for the diffusion process to work, although Baschek and Norris (1970) find that the subdwarf HD 205805 has a surface helium deficiency of 10, while Sargent and Searle's data suggest even greater deficiences for three sdB halo stars.

\section{Individual, Old Binary Systems; $\mu$ Cas and 'a Recent Determination of Primordial Helium Abundance'}

One reason for the interest in helium contents of stars, particularly old stars, is of course that such knowledge may give us valuable information about the early composition of our Galaxy and, presumably, the preceding era. To this end, one can search for well-determined unevolved subdwarf binary systems. Such systems are so rare as to be almost non-existent. However, a flurry of excitement occurred among cosmologists earlier this year when Hegyi and Curott (1970) claimed to have determined an upper limit on the primordial helium abundance by applying theoretical ML relations (Faulkner, 1967) to new observations of $\mu$ Cas. Because of the interest expressed in this result, I have subjected it to a careful examination. I present my conclusions as a case history for caution.

$\mu$ Cas, one of the nearest and brightest mild subdwarfs, has a fairly well-determined photocentric orbit (Lippincott and Wyckoff, 1964), but attempts at visual resolution have been either unsuccessful or ambiguous. Wehinger and Wyckoff's (1966) observations led to a primary mass of $\sim 0.75 M_{\odot}$, which was then used by Catchpole et al. (1967) to deduce a helium abundance little different from that of the Sun, namely $Y=0.26$. This should however be treated with extreme scepticism since Wehinger and Wyckoff's observed position angle was at right angles to that predicted by the orbit!

The novel approach suggested by Hegyi and Curott was to chop the combined stellar image and, by rapid scanning on a msec time-scale, to 'freeze' the effects of seeing. During a typical observation some 20000 scans were made with the hope of producing, statistically, a good separation. This is an exciting observational development, typical of the experimental cunning of Princeton's Palmer Physical Laboratory, and one hopes the approach may become a standard tool for double star observers.

Unfortunately, the results in the present application contain some difficulties for interpretation. The equipment was calibrated against known (but presumably widely separated) binaries for one direction of scanning, while the observations on $\mu$ Cas were conducted in both this and the reverse direction. The latter results apparently differed significantly from the former, which were taken as the final observations in view of the calibration scanning direction being the same. Even so, the distribution of results appeared far from normal so that my own feeling is that the observed separation, $1^{\prime \prime} .09 \pm 0 \prime 10(\sigma)$ may have a much larger probable error than the formal 
standard deviation would suggest. On the basis of Lippincott and Wyckoff's photocentric orbit, Hegyi and Curott claim that the mass of $\mu$ Cas $\mathrm{A}$, in solar units is $1.54_{-0.42}^{+0.47}$. By appealing to quasi-homology relations (Faulkner, 1967) they deduced a negative helium abundance, with $1 \sigma$ and $2 \sigma$ internal limits permitting $Y \sim 0.05$ and $Y \sim 0.34$.

To an astronomer a mass this high seems uncomfortably at variance with the knowledge that $\mu$ Cas is classified as G5 VI, i.e. a subdwarf three subclasses later than the Sun. In order to check the mass a colleague, K. Kamper, and I, in independent calculations, concluded that $\mu$ Cas A has a mass of $\sim 1.37 M_{\odot}$ using the Hegyi and Curott separation. The only way of obtaining $\sim 1.54 M_{\odot}$ appears to involve the use, in Kepler's law, of the observed separation of 1".09 rather than the semi-major axis of the true relative orbit, which we determine to be equivalent to $\sim 1$ 1".04 at this distance. Thus it would unfortunately appear that the sophistication of the experiment is not matched by that sophistication of analysis which we have come to expect from this laboratory.

Assuming that the remaining analysis is correct, the mass of $\sim 1.37 M_{\odot}$ again implies $Y<0$, but this time the $1 \sigma$ and $2 \sigma$ limits permit $Y \sim 0.2$ and $Y \sim 0.45$, which results seem wide enough to encompass any desired conclusion.

Other possible errors which have not been considered are due to uncertainties in the orbit itself. The new observations, made in August 1967, occurred over $4 \frac{1}{2} \mathrm{yr}$ after the latest observations (January 1963) going into the orbit determination. Wagman (1961) obtained a provisional period of $23 \mathrm{yr}$, while Lippincott and Wyckoff obtained $18.5 \mathrm{yr}$. According to the orbit, the separation would be rapidly closing, and a $10 \%$ change would occur in 6-7 months. Dr Lippincott has kindly informed me that in view of possible uncertainties remaining both in the period and in the epoch of periastron passage, an error corresponding to this amount is still possible.

Before leaving these considerations entirely, it may perhaps be useful to point out the error $\Delta Y$ in deduced helium abundance which corresponds to errors of say $10 \%$ in the semi-major axis $a$ of the relative orbit. These results have been obtained from quasi-homology relations (Faulkner, 1967) which, though recently criticized, agree reasonably well with the work of others:

\begin{tabular}{ll} 
True $Y$ & $\Delta Y(\Delta a / a=0.1)$ \\
\hline 0.00 & 0.28 \\
0.25 & 0.25 \\
0.50 & 0.22
\end{tabular}

Thus the actual error in the deduced helium content is almost three times the fractional error in the semi-major axis - a very strong dependence which is of course implicit in the $1 \sigma$ and $2 \sigma$ limits quoted above. If one wishes to know $Y$ to within 0.10 , it is necessary to know the semi-major axis to within 3 or $4 \%$. Similar remarks apply to the required accuracy of the parallax. 


\section{Post Main-Sequence Evolution; the Shape of the Sub-Giant Branch}

In 1966, following up implications of a theory of the horizontal branch (Faulkner, 1966), Iben and I found (Faulkner and Iben, 1966) that, all other things being equal, the subgiant branch computed for metal weak models was much steeper for high helium content than for low helium content. It was already well-known that the almost horizontal subgiant branches of old Population I clusters (e.g. M 67, NGC 188 ) could be matched with high helium and relatively normal metal content. However, attempts to match the globular cluster subgiant branches with both low helium and low metals had never succeeded. With our work, it became possible to contemplate a situation in which the major difference between the clusters exhibiting vastly different subgiant slopes was one of metal content alone (with possibly minor differences in helium content and age). Furthermore, this economy of hypotheses required that the commonly shared helium abundance be fairly substantial. (This latter deduction may, of course, be treated as an additional hypothesis by those hostile to the result.)

However, all other things are rarely equal, and a given shape may be matched by variations in some or all of helium content, metal content, mixing length, and age. . In particular, Iben and Rood (1970a) recently demonstrated that similar shapes were obtainable for various sequences with $Y=0.20$ and 0.30 by varying the heavy element content $Z$ in the range $10^{-3}-10^{-4}$, and ages by a factor of 2 . My impression is that it would still be difficult to match the shapes with $Y \lessgtr 0.10$. However, in view of uncertainties in mixing length, and the Iben and Rood results, satisfactory matching of the subgiant shape should be regarded only as a brick, rather than a foundation stone, in a theory deducing the helium abundance of globular cluster stars.

\section{The Horizontal Branch and Later Stages}

By the early 1960's, attempts were made to construct models for horizontal branch stars, for example by Japanese workers (see Hayashi et al., 1962). They considered a low helium model which, having thrown off a large mass fraction at the end of the giant phase, evolved across the horizontal branch from blue to red. In 1966 I advanced a theory in which, though such models were possible, it was shown that the observed correlations of horizontal branch characteristics (e.g. 'gaps') with metal content were more readily understood if the helium content were high. I remarked at that time that, with helium core-masses having been established by evolution up the giant branch, variable amounts of envelope mass-loss would be an obvious way of populating the horizontal branch of a given cluster at any one time. Recent theoretical and observational work would appear to require such mass-loss.

However, at that time I also remarked that mass loss need not be an essential requirement of the theory owing to the curious circumstance that low helium envelopes produced negligible hydrogen shell sources, while high helium envelopes had important hydrogen shell sources. The former models would then evolve like core- 
burning stars, i.e. to the red (as shown by Hayashi et al.), while there seemed a reasonable chance that the latter would evolve to the blue, as was known to occur for more massive stars in similar circumstances. Investigations with Iben (Faulkner and Iben, 1966) indeed showed this to be the case.

Nevertheless, early observational difficulties were produced for the high helium picture by, among others, Searle and Rodgers (1966), Sargent and Searle (1966) and Greenstein père and Münch (1966). These authors showed that there were many examples of blue halo stars (thought to be horizontal branch stars), and also blue $\mathrm{HB}$ stars in observed globular clusters, with abnormally weak helium lines and, presumably, low helium content. However, the helium deficiences claimed (factors of 50-100 lower than the Sun) soon led to a curious reductio ad absurdum. In an apparently little known letter to Nature, Faulkner and Iben (1967) showed that the long climb of a star up the red giant branch is accompanied by helium enrichment of the outer convective envelope. This envelope descends deep into the star and (if I may encroach, giant-like, upon Dr. Kippenhahn's helium production preserves), dredges up helium from the varying profile established during the main sequence phase. For $Y_{0}=0,0.10$ and 0.35 the enrichment is $\Delta Y \sim 0.025,0.015,0.005$. Similar results have since been obtained by Henyey (1970) and Demarque (1970). Thus the minimum helium content which should hold throughout at least the sub-photospheric layers, and very probably the photosphere too, is $\sim 2.5 \%$, i.e. $\sim \frac{1}{10} Y_{\odot}$. In finding a deficiency factor of 50-100, the observers are hoist on their own petard, having established that spectroscopic observations, at least for such advanced phases, are not relevant to material below the immediate photospheric layers.

A suggestion to this effect by Greenstein fils et al. (1967) had already appeared in the literature. Their conjectured mechanism, involving diffusion and gravitational settling of helium, has received additional support recently (albeit obliquely) from work by Michaud (1970) as mentioned earlier. Additional evidence for surface separation comes from Sargent and Searle (1967) who found the field HB star, Feige 86, to have spectrum peculiarities similar to certain rare Population I B stars with weak helium lines in circumstances strongly suggesting that the latter cannot be representative of the interior. I remind you once more of the sharplined weak helium B stars in Orion in which, as they lie along the lower edge of the main-sequence, the helium deficiency cannot, according to stellar structure, extend throughout the interior. One is mindful of a remark attributed to Lyttleton - "If one thinks one can determine the distribution of the elements inside stars from their surface composition, one may as well believe a chimney sweep is made of solid carbon."

There has always been however a more serious problem for the picture of zero mass-loss in HB stars. It was difficult to obtain the observed lengths (in $\log T_{\mathrm{e}}$ or equivalently B-V) of horizontal branches. Furthermore, each modernization of the equations of state, surface programs and opacities had the disturbing consequence of moving all tracks to the red and shortening them still further. Thus, although it was possible (Iben and Faulkner, 1968) to obtain a good, self-consistent zero massloss fit to many properties of globular clusters (giant branch shapes, relative bright- 
ness of horizontal branch and main-sequence turn-offs, etc.), the blue-ward evolution was never of a sufficiently large extent. In spite of this, a possible observational test (Hartwick et al., 1968) appeared to give marginal support to normal helium content for M 92. The same test applied to NGC 6397 (Newell et al., 1969) gave results of doubtful interpretation. Recently, Iben and Rood (1970b) have bowed to the inevitable and accepted that a certain amount of mass loss may be required.

To return to the question of helium abundance, a new type of comparison, first used by Iben (1968a) for M 15, appears extremely promising, if not conclusive. Iben et al. (1969) have recently compared the ratio $N_{\mathrm{HB}} / N_{\mathrm{RG}}$ for the seven globular clusters in Arp's classic study (Arp 1955). Here $N_{\mathrm{HB}}$ and $N_{\mathrm{RG}}$ are the numbers of stars on the horizontal branch and red giant branch respectively. The ratio should be close to the theoretically computed lifetime ratio, $t_{\mathbf{H B}} / t_{\mathrm{RG}}$. To a good approximation, the lifetimes in these two phases are determined by the core masses and envelope compositions the lifetime in the horizontal branch phase is almost independent of the amount of envelope mass-loss. From theory, $t_{\mathrm{HB}} / t_{\mathrm{RG}}$ varies from $\sim 0.4(Y \sim 0.10)$ to $\sim 1(Y \sim 0.30)$. The total observed range found by Iben et al. is $N_{\mathrm{HB}} / N_{\mathrm{RG}} \sim 0.80-0.96$. They conclude that for these globular cluster stars, $Y \gtrsim 0.25$.

One cannot, of course, discuss the horizontal branch without referring to the RR Lyrae variables and Christy's monumental paper (Christy, 1966) on their properties. Time does not permit me to do other than briefly mention his conclusions concerning the helium content, which remained essentially unchanged in his later paper (Christy, 1968). Christy's requirements for helium to drive his pulsations are perhaps expressed more cautiously by him than by others who quote him. Nevertheless, he would find it difficult to excite the required pulsations for $Y \leqslant 0.15$. Furthermore he finds that for M 3 the shapes of light- and velocity-curves best fit his models for $M \simeq 0.5 M_{\odot}$. With luminosity deduced from the shortest observed fundamental period, and using the distribution of first harmonic and fundamental pulsators, he obtains a probable range for helium abundance of $\sim 25-40 \%$ in $\mathrm{M} 3$. A possibly more accurate determination comes from a comparison of the high temperature edge of the instability strip with his models, which yields $Y \sim 30 \%$. At a meeting of Commission 35 , Iben stated that he has found the position of this boundary to be sensitive to the inclusion of radiation pressure in the calculations, to the tune of several hundred degrees. Iben claims a fit to $\mathrm{M} 3$ when including radiation pressure (apparently omitted by Christy) for $Y \sim 0.26$ so that the disagreement, though important in principle, is relatively small as far as the deduced helium content is concerned.

An additional indirect piece of evidence for high $Y$ is the work of Schwarzschild and Härm (1970) on the evolutionary phase of Cepheids in globular clusters. Of several post-horizontal branch models which they evolve, only those with $Y=0.30$ pass, in thermal pulse phases, through the region of the HR diagram occupied by Population II Cepheids. Models with $Y=0.10$ or 0.00 keep stubbornly to the giant branch. This may in part be a consequence of the masses chosen for these models, which would correspond to the similar ability of the HB stars themselves to become detached from the giant branch (Faulkner, 1966). In any event, Wallerstein (1970) 
has pointed out that globular cluster Cepheids only occur in clusters with a blue horizontal branch, a correlation consistent with the high helium $(Y=0.30)$ and low masses $\left(0.65 M_{\odot}\right)$ for which Schwarzschild and Härm find that excursions from the giant branch occur.

\section{The Solar Helium Content}

We come finally to a more parochial problem, the helium content of the solar interior. This has been discussed lately ad nauseam in connection with the search for solar neutrinos (Davis et al., 1968). I shall therefore limit my remarks to the main advances and retreats leading up to the status quo.

Honesty compels one to say at the outset that the upper limit to the solar neutrino flux obtained by Davis et al. sets the theory of stellar interiors yet one more great problem. The original upper limit, based essentially on a null result in comparison with background counts, was expressed as

$$
\sum \phi_{i} \sigma_{i} \leqslant 3 \times 10^{-36} v \text {-captures per sec per }{ }^{37} \mathrm{Cl} \text { atom, }
$$

where $\phi_{i}$ is the flux of neutrinos at the Earth from the $i$ th neutrino-producing solar nuclear reaction, and $\sigma_{i}$ is the integrated capture cross-section for such neutrinos. There is some indication (Shaviv, 1970; Cameron, 1970) that with improved techniques the upper limit is now turning into a formal result, expressed as

$$
\sum \phi_{i} \sigma_{i}=(2 \pm 1) \times 10^{-36} v \text {-captures per } \sec \text { per }{ }^{37} \mathrm{Cl} \text { atom }
$$

For purposes of discussion and comparison, I shall introduce a new unit, the SNAFU (solar neutrino approximate flux unit), equal to $2 \times 10^{-36} v$-captures per sec per ${ }^{37} \mathrm{Cl}$ atom. Thus the result currently attributed to Davis may be expressed as $1 \pm 0.5$ SNAFU.

I now turn to the solar models themselves. The modern era of solar models began with that of Sears (1964). His preferred model $J$ included, as input, a metal to hydrogen ratio (by mass), of $Z / X \sim 0.028$, obtained from a judicious combination (Gaustad, 1964) of photospheric and solar cosmic ray rocket data. The resulting value of the helium content required to fit the solar luminosity at an age of $\sim 4.5 \times 10^{9}$ yr was $Y \sim 0.27$. However, the neutrino counting rate was equivalent to approximately 14 SNAFU (the non-preferred models giving up to 30 SNAFU).

Subsequent changes in the physical data prior to the Davis result included several nuclear cross-section factors (affecting in particular the rates of the ${ }^{7} \mathrm{Be}+p$ and ${ }^{3} \mathrm{He}+{ }^{3} \mathrm{He}$ reactions), the neutron lifetime (increasing the $p+p$ reaction rate and thus lowering the central solar temperature), and the photospherically deduced $Z / X$, which was reduced to $\sim 0.019$ by Lambert and Warner (Lambert 1968a, b; Lambert and Warner 1968a, b, c, d; Warner, 1968). Thus at the time the result of Davis et al. was published, Bahcall et al. (1968) had obtained a neutrino capture rate of $3.8 \pm 1.5$ SNAFU, and solar helium content, $Y \sim 0.22 \pm 0.03$. Iben $(1968 \mathrm{~b}, 1969)$ objected strongly to the claim that this result was not irreconcilable (because of parameter 
uncertainties) with the experiment, and pointed out that strict adherence to physical data and the neutrino counts required $Y \lesssim 0.16-0.17$. He showed that the higher value was possible only if one or more of a large variety of physical data and assumptions were in error by much more than the commonly quoted probable error limits.

In most models, $80-90 \%$ of the predicted counts come from neutrinos produced by the decay of ${ }^{8} \mathrm{~B}$, which is itself produced only in a rare, highly temperaturesensitive sub-branch of the $p p$-chain (in fact, $\phi_{v}\left({ }^{8} \mathrm{~B}\right) \propto T_{c}^{14}$ ). For this reason a lot of effort has been put into ways of reducing the solar central temperature, $T_{c}$. The literature has been rapidly filling with ingenious suggestions to effect this, few of which seem particularly satisfactory or efficacious. Since the problem is to lower $T_{c}$, methods of reducing the current mean molecular weight have claimed much attention. These have included the possibility of long-lived convective cores following the Hayashi phase or, for example, the mixing of hydrogen-rich material into the depleted central regions, which might occur if the Sun possessed a rapidly spinning core. Shaviv (1970), by comparison with observed properties of low-mass globular cluster stars, has deduced a mixing limit which for a solar model permits perhaps a $40 \%$ reduction in the predicted neutrino counts.

Attempts to lower $T_{c}$ have however been largely frustrated by recent increases in the net solar opacity, arising from the combined effects of electron correlations (decreased opacity; Watson, 1969b), autoionization (increased opacity; Watson, 1969a) and the upward revision of the solar iron abundance by a factor of $\sim 10$ (Garz et al., 1969). Whereas a further reduction in the ${ }^{7} \mathrm{Be}+p$ cross-section factor had led to a counting rate of 3 SNAFU (Bahcall et al. 1969), Watson (1969c) suggested that the combined opacity increases would add about $30 \%$ to this figure. This was confirmed in detail by Bahcall and Ulrich (1970) who found, when including all the above changes, a count of 3.9 SNAFU of which 0.85 SNAFU come from neutrino producing reactions other than ${ }^{8} \mathrm{~B}$ decay. Although Bahcall and Ulrich are now moved to admit that a sizable discrepancy exists between theory and experiment, there is still a faint hope that the blame can be laid, somehow, on the rare ${ }^{8} \mathrm{~B}$ mode of $p p$-chain completion.

Because certain observables are fixed for the Sun (e.g. mass, present luminosity, present radius), it is easy to show that an increase in opacity not only causes an increase in $T_{c}$ (and hence $\phi_{v}$ ) but also requires, via the virial theorem, an increase in mean molecular weight. Thus one can qualitatively understand the latest helium figure of Bahcall and Ulrich, $Y \sim 0.26$.

We are still left with the dilemma pointed out by Iben, if we wish to obtain a total count of only 1 SNAFU. This has been confirmed by Shaviv (1970), whose results I have summarized in the following table. In the table, $L \& W$ represents (with the exception of the iron abundance) Lambert and Warner values for $Z / X$, while 'old' and 'new' entries in the iron abundance column refer to $\log N_{\mathrm{Fe}}=6.6$ and 7.6 respectively $\left(\log N_{\mathrm{H}} \equiv 12.00\right)$. The final row is obtained by assuming that the relative heavy element abundances (except iron) are inter alia those of Lambert and Warner but that $Z / Y$ is fixed by solar cosmic ray data. 


\begin{tabular}{lllr}
$Z / X$ & Fe & $Y$ & SNAFU Count \\
\hline$L \& W$ & New & 0.26 & $\sim 3.5$ \\
$L \& W$ & Old & 0.21 & $\sim 1.9$ \\
$\sim 0.6(L \& W)$ & New & $0.15-0.16$ & 1.0
\end{tabular}

Thus the final row requires that the photospheric $Z / X$ be lower than Lambert and Warner's value by a factor even larger than that involved in changing from Gaustad's to Lambert and Warner's result. Lambert and Warner's quoted uncertainties are generally of order one quarter to one half of the required systematic reduction of $\sim 0.2$ in $\log Z / X$. An alternative way of putting this conclusion is to assert that, for some unknown reason, contributions of the heavy elements to the opacity are too large by factors of maybe 1.5-2; or, opacities generally, including (heaven forbid!) contributions from hydrogen and helium, are all too large by lesser factors.

\section{Summary}

We seem to have come full circle, ending, as we began, with the suspicion that stellar opacities may be responsible for many of our unsolved problems. While this remains a possibility it is certainly premature to draw firm conclusions about the helium contents of stellar interiors. However, to throw caution aside, let me state once again that $I$ believe there is no requirement from stellar interiors for essentially zero helium. Indeed, the only requirement for $Y \leqslant 0.2$ comes from the solar neutrino counts (perhaps a powerful requirement nonetheless) with a large reduction in $Z / X$ or computed opacities necessary for complete consistency. Lower opacities generally would help reduce the large helium content required to explain the Hyades observations, though we believe the latter are not unassailable. Schwarzschild (1970) has pointed out the danger of accepting present opacities as final, in particular for the horizontal branch where lengths of evolutionary tracks are enormously sensitive. It may well be that our subject matter awaits a saviour who can make it less opaque.

\section{Acknowledgements}

I am indebted to the many astronomers with whom I have discussed various aspects of the helium problem. In particular, I thank D. Butler for a most thorough search of the literature; the blame for errors, be they of omission or commission, rests entirely upon my shoulders. This work was supported in part by a National Science Foundation Grant No. GP-11142.

\section{References}

Alexander, J. B.: 1968, Quart. J. Roy. Astron. Soc. 9, 136.

Arp, H. C.: 1955, Astron. J. 60, 317. 
Bahcall, J. N., Bahcall, N. A., and Shaviv, G.: 1968, Phys. Rev. Letters 20, 1209.

Bahcall, J. N., Bahcall, N. A., and Ulrich, R. K.: 1969, Astrophys. J. 156, 559.

Bahcall, J. N. and Ulrich, R. K.: 1970, Astrophys. J. 160, L57.

Baschek, B. and Norris, J.: 1970, Astrophys. J. Suppl. No. 176 19, 327.

Bodenheimer, P. H.: 1965, Astrophys. J. 142, 451.

Cameron, A. G. W.: 1970, Commission 35 discussion, IAU.

Catchpole, R. M., Pagel, B. E. J., and Powell, A. L. T.: 1967, Monthly Notices Roy. Astron. Soc. 136, 403.

Cayrel, R.: 1968, Astrophys. J. 151, 997.

Christy, R. F.: 1966, Astrophys. J. 144, 108.

Christy, R. F.: 1968, Quant. J. Roy. Astron. Soc. 9, 13.

Davis, R. Jr., Harmer, D. S., and Hoffmann, K. C.: 1968, Phys. Rev. Letters 20, 1205.

Demarque, P.: 1968, Astron. J. 73, 669.

Demarque, P.: 1970, private communication.

Eggen, O. J.: 1962, Quant. J. Roy. Astron. Soc. 3, 259.

Eggen, O. J.: 1963, Astrophys. J. Suppl. No. 76, 8, 125.

Eggen, O. J.: 1969, Astrophys. J. 156, 241.

Eggen, O. J. and Sandage, A. R.: 1962, Astrophys. J. 136, 735.

Faulkner, J.: 1964, Ph. D. thesis, Univ. of Cambridge, England.

Faulkner, J.: 1966, Astrophys. J. 144, 978.

Faulkner, J.: 1967, Astrophys. J. 147, 617.

Faulkner, J. and Iben, I., Jr.: 1966, Astrophys. J. 144, 995.

Faulkner, J. and Iben, I., Jr.: 1967, Nature 215, 44.

Garz, T., Kock, M., Richter, J., Baschek, B., Holweger, H., and Unsöld, A.: 1969, Nature 223, 1254.

Gaustad, J.: 1964, Astrophys. J. 139, 406.

Greenstein, G. S., Truran, J. W., and Cameron, A. G. W.: 1967, Nature 213, 871.

Greenstein, J. L. and Münch, G.: 1966, Astrophys. J. 146, 618.

Hartwick, F. D. A., Härm, R., and Schwarzschild, M.: 1968, Astrophys. J. 151, 389.

Hayashi, C., Hoshi, R., and Sugimoto, D.: 1962, Progr. Theoret. Phys. Suppl. No. 22, 1.

Hegyi, D. and Curott, D.: 1970, Phys. Rev. Letters 24, 415.

Henyey, L. G.: 1970, private communication.

Hodge, P. W. and Wallerstein, G.: 1966, Publ. Astron. Soc. Pacific 78, 411.

Hubbard, W. B. and Lampe, M.: 1969, Astrophys. J. Suppl. No. 163, 18, 297.

Iben, I., Jr.: 1967, Ann. Rev. Astron. Astrophys. 5, 600.

Iben, I., Jr.: 1968a, Nature 220, 143.

Iben, I., Jr.: 1968b, Phys. Rev. Letters 21, 1208.

Iben, I., Jr.: 1969, Ann. Phys. 54, 164.

Iben, I., Jr. and Faulkner, J.: 1968, Astrophys. J. 153, 101.

Iben, I., Jr. and Rood, R.: 1970a, Astrophys. J. 159, 605.

Iben, I., Jr. and Rood, R.: 1970b, Astrophys. J. 161, 587.

Iben, I., Jr., Rood, R., Strom, K. M., and Strom, S. E.: 1969, Nature 224, 1006.

Lambert, D. L.: 1968a, Observatory 87, 228.

Lambert, D. L.: 1968b, Monthly Notices Roy. Astron. Soc. 138, 143.

Lambert, D. L. and Warner, B.: 1968a, Ibid. 181.

Lambert, D. L. and Warner, B.: 1968b, Ibid. 213.

Lambert, D. L. and Warner, B.: 1968c, Ibid. 229.

Lambert, D. L. and Warner, B.: 1968d, Monthly Notices Roy. Astron. Soc. 140, 197.

Lippincott, S. L. and Wyckoff, S.: 1964, Astron. J. 69, 471.

Michaud, G.: 1970, Astrophys. J. 160, 641.

Newell, E. B., Rodgers, A. W., and Searle, L.: 1969, Astrophys. J. 156, 597.

Norris, J.: 1970a, Astrophys. J. Suppl. No. 176 19, 337.

Norris, J.: 1970b, private communication.

Popper, D. M., Jørgensen, H. E., Morton, D. C., and Leckrone, D. S.: 1970, Astrophys. J. 161, L57.

Sargent, W. L. W. and Searle, L.: 1966, Astrophys. J. 145, 652.

Sargent, W. L. W. and Searle, L.: 1967, Astrophys. J. 150, L33.

Sargent, W. L. W. and Searle, L.: 1968, Astrophys. J. 152, 443. 
Sargent, W. L. W. and Strittmatter, P. A.: 1966, Astrophys. J. 145, 938.

Schwarzschild, M.: 1970, Remarks at the 132nd meeting of the American Astronomical Society, Boulder, Colorado, U.S.A.

Schwarzschild, M. and Härm, R.: 1970, Astrophys. J. 160, 341.

Searle, L. and Rodgers, A. W.: 1966, Astrophys. J. 143, 809.

Sears, R. L.: 1964, Astrophys. J. 140, 477.

Shaviv, G.: 1970, Commission 35 discussion, IAU.

Strom, S. E. and Strom, K. M.: 1967, Astrophys. J. 150, 501.

Wagman, N. E.: 1961, Astron. J. 66, 433.

Wallerstein, G.: 1970, Astrophys. J. 160, 345.

Warner, B.: 1968, Monthly Notices Roy. Astron. Soc. 138, 229.

Watson, W. D.: 1969a, Astrophys. J. 157, 375.

Watson, W. D.: 1969b, Astrophys. J. 158, 303.

Watson, W. D.: 1969c, Ibid. L189.

Wayman, P. A.: 1967, Publ. Astron. Soc. Pacific 79, 156.

Wayman, P. A., Symms, L. S. T., and Blackwell, K. C.: 1965, Roy. Obs. Bull. No. 98.

Wehinger, P. A. and Wyckoff, S.: 1966, Astron. J. 71, 185.

\section{DISCUSSION}

D. M. Popper: Despite Professor Tayler's omission of the mass-luminosity relation as a method of evaluating the helium abundance in stellar interiors, it may be pertinent to refer to results from the best eclipsing binaries. (At this point, two slides were shown: the mass-ratios and mass-luminosity relations as appearing in Popper, Jørgensen, Morton, and Leckrone, 1970, Astrophys. J. 161, L57.) The systems shown are uncomplicated ( $A$ and $F$ type) main-sequence systems. The evolved systems are clearly shown as having the larger radii for a given mass. Adoption of a temperature scale enables the luminosities of the systems to be evaluated. For a heavy element abundance, $Z=0.03$, the positions of components of the seven apparently unevolved systems in the mass-luminosity plane led to a number ratio $N(\mathrm{He}) / N(\mathrm{H})=0.12$. An iron abundance about 5 times the Goldberg-Müller-Aller value is adopted in the opacities in obtaining this result. The limits on $N(\mathrm{He}) / N(\mathrm{H})$ of 0.095 and 0.135 take into consideration uncertainties in both observational and theoretical factors. It is reasonable to expect the number of systems with good masses and radii in the range late $B$ to early $G$ will be approximately doubled in the not-too-distant future, particularly if the photometric observations of these systems are pushed vigorously.

John Faulkner: Thank you. This is, as far as I am aware, the first time that a careful analysis of the observations shows the theoretically predicted change in slope in the $M-L$ relation from $\approx 5$ for $1 M_{\odot}$ to $\approx 4$ for $2.5 M_{\odot}$.

R.J.Tayler: In reply to Dr. Popper, I failed by accident to mention the mass-luminosity relation as a method for determining helium abundance. Clearly, it is important: if we cannot understand the structure of main sequence stars, we cannot hope to understand the structure of evolved stars. The crucial question, however, is whether a better result is obtained from the $M / L$ relation using a few reliable stars or from the shape of an entire cluster HR diagram.

Icko Iben, Jr: (1) Using the 'best' input physics, model estimates of the Sun's helium abundance give $Y \approx 0.25-0.26$, not $Y \approx 0.20$ as suggested by Dr. Tayler. On the other hand, the 'best' solar models also give a flux of $B^{8}$ neutrinos that is from 3 to 6 times larger than Davis's current upper limit. In order to achieve a neutrino flux consistent with Davis's limit, it is necessary to assume that relevant opacities are too large by a factor of $2-4$; then solar models have $Y \approx 0.15$.

(2) Dr. Tayler suggested that potentially the 'best' way to determine the $Y$ for cluster stars is to compare models evolving from the main sequence to the giant branch with stars on the subgiant portion of cluster diagrams. Such a comparison is in fact the worst one to focus on. This is because theoretical results are extremely sensitive to the treatment of convection in the envelopes of stars evolving beyond the main sequence. As is well known, a reliable treatment of convection does not exist.

(3) The last word has not yet been given on estimates of $Y$ from a comparison between theoretical and observational estimates of the blue edge of the instability strip. As Christy has shown, the location of the blue edge depends on the assumed value for $Y$. Larger $Y$ means a larger $\log T_{\text {eff }}$ at the blue 
edge. Using Christy's published (1966) results and estimating unreddened colors for the blue edge in M15, M92, and M3, Sandage suggests $Y \approx 0.32$.

I have constructed a linear non-adiabatic program to trace down more carefully the dependence of the blue edge on various theoretical input parameters. One result of a survey is that, for a given $Y$ in the neighborhood of 0.3 , the blue edge is bluer than that found by Christy by $\Delta\left(\log T_{\mathrm{epr}}\right)=0.02$. The sensitivity of $\log T_{\text {eff }}$ at the blue edge to changes in $Y$ is larger than that found by Christy by about a factor 2 . Specifically, the blue edge becomes bluer by $\Delta\left(\log T_{\text {eff }}\right) \approx 0.02$ for an increase in $Y$ of about 0.06 .

Thus, adopting Sandage's treatment of the data, one obtains $Y \approx 0.26$ for RR Lyrae stars in M15, M92, and M3.

R.J. Tayler: I hope that I said that the use of the lower part of a cluster HR diagram may not give very good values for the helium abundance, but it is nevertheless very important because it deals with old unevolved stars.

P. Giannone: I would like to report that according to work by Castellani, Renzini and myself on horizontal branch stars, an intermediate semiconvective zone has been encountered in the evolutionary calculations. The occurrence of this intermediate zone is related to the behaviour of the opacity values for carbon-oxygen-rich and helium-rich mixtures. An increase in mass of the convective core during the initial phase of central helium burning induces a partial mixing in the region outside the core. In a star model of $0.7 M_{\odot}\left(X_{e n v}=0.9, Z=0.001\right)$ with an initial mass-fraction in the helium core equal to 0.9 , the semi-convective zone appears when the central helium abundance is decreased to about 0.7 . At the end of the central helium burning, the star model consists of a carbon-oxygen core containing a mass-fraction of about $20 \%$, an intermediate semi-convective region with a varying chemical composition of about $25 \%$ in mass, and a radiative envelope. The lifetime of the central helium burning and the corresponding extension of the evolutionary track along the horizontal branch are almost doubled with regard to the evolutionary track obtained with a step-profile in the chemical composition at the core boundary.

D. M. Popper: I would like to summarize some work by R. K. Ulrich on 'Possible evidence of $\mathrm{He}^{3}$ in the color-magnitude diagram of NGC 2264'.

A moderate initial abundance of $\mathrm{He}^{3}$ has a large effect on the structure of a star because the crosssection for the reaction $\mathrm{He}^{3}+\mathrm{He}^{3} \rightarrow \mathrm{He}^{4}+2 \mathrm{H}^{1}$ is a factor $10^{10}$ greater than the cross-section for the $p-p$ reaction in the center of a two-solar mass zero-age main-sequence star. Models constructed with an initial $\mathrm{He}^{3}$ mass fraction of $0.5 \%$ have a central temperature $40 \%$ lower than that for zero-age main-sequence models without $\mathrm{He}^{3}$ and lie in a region of the color-magnitude diagram where gravitationally contracting stars are usually found. There is another factor which is unique to $\mathrm{He}^{3}$ : the $\mathrm{He}^{3}-\mathrm{He}^{3}$ reaction increases the number of particles per gram and hence decreases the mean molecular weight. A gradient of molecular weight opposite in sign to the temperature and density gradients leads to local mixing, and it seems reasonable to postulate that stars burning $\mathrm{He}^{3}$ are completely mixed. The additional fuel available with th is postulate increases the $\mathrm{He}^{3}$ burning lifetime by a factor of 10 .

The slide shows the well-known color-magnitude diagram of the young cluster NGC 2264 by Walker.

The problem is that stars of $(\mathrm{B}-\mathrm{V}) \approx 0.6$ are too close to the zero age main sequence to be compatible with the age of about 3 million years implied by the main sequence arrival point near $(B-V)=0.0$. Although the inclusion of the Hayashi convective phase has removed some of the problem, there are many stars in the cluster whose masses are between 1.2 and 1.5 solar masses and which are found too close to the main sequence to be only 3 million years old. Modern calculations by Iben or by Kippenhahn and Weigert show that it takes about 15 to 20 million years for these stars to arrive near the sequence. The suggestion is made that the estimate of $15-20 \times 10^{6}$ years is correct and that the arrival of the higher mass stars $(B-V \approx 0)$ on the main sequence is delayed by the presence of $\mathrm{He}^{3}$. The slide shows the isochrones of Kippenhahn and Weigert for $3 \times 10^{6}$ years, with no $\mathrm{He}^{3}$, as well as for a mass-fraction of $\mathrm{He}^{3}=0.008$ and an age of $15 \times 10^{6}$ years.

A. G. Massevitch: When comparing results of model computations with observed positions in the $\mathrm{H}-\mathrm{R}$ diagram, the most dangerous operation is the transition from theoretical quantities $(L, R)$ to observed ( $M_{v}, T_{\text {eff }}$, or colours). The application of different temperatures scales leads to a rather substantial dispersion in calculated helium abundances and to an even larger spread in bolometric corrections. The discrepancies are in some cases larger than those caused by differences in opacities. Authors should be urged to mention what bolometric corrections and temperature scales have been 
used when publishing results of computations. It seems high time that there should be a general agreement established between observers and theoreticians as to what $T$-scales and B.C's should be applied for transferring theoretical values into observed.

$R$. Schwartz: What is the effect of the discrepancy between the pulsation masses and the evolutionary masses on the calculated helium abundances in Cepheids and RR Lyrae stars?

R. F. Christy: Discrepancies between the masses that fit pulsation calculations to observation and the masses from evolutionary theory lead me to seriously doubt the basic models used and thereby the opacities on which they depend. If the opacities are very significantly altered, we would expect the calculated $T_{\text {efr }}$ of the blue edge of the instability strip to be altered. Thus the deduction of the helium abundance $Y \sim 0.3$ in the envelope from this becomes more doubtful.

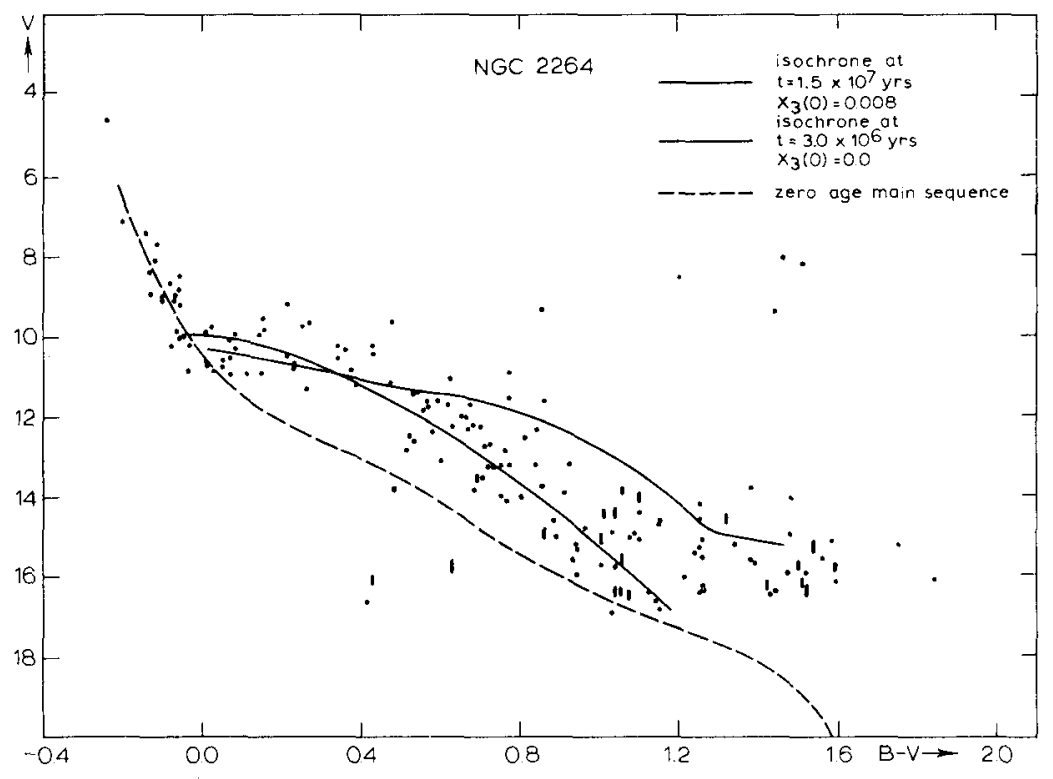

Fig. 2. Color-magnitude diagram of the young cluster NGC 2264 by Walker.

$N$. H. Baker: It has been remarked that the method of determining the He abundance, $Y$, by computing the effective temperature of the blue edge of the RR Lyrae instability strip and comparing it with the blue edge of the gap, is subject to uncertainties in the opacities. Further evidence for this is provided by a recent study by T. J. van Albada and myself. On the basis of the linear non-adiabatic theory of pulsation, we find that with Christy's (1966) parameters and opacities, the maximum temperature $T_{\text {efr }}$ (max) of unstable stars (in the first overtone) as a function of $Y$ agrees well with Christy's results (to within $100^{\circ}-150^{\circ}$ ). If, on the other hand, we use the Cox-Stewart opacities used by Iben and Rood (1970) we find a considerable deviation from Christy's results. For a given mass, luminosity and heavy-element abundance $(Z)$, the two relations are plotted on the accompanying graph, (Figure 3 ) (our relation also depends slightly on envelope convection; $l / H_{p}$ is the ratio of mixing lengths to pressure scale height).

The main point is that this relation is in fact very sensitive to the opacities used, especially since the stability depends strongly on the variation of opacity with temperature and density in the stellar envelope. Furthermore, if the dependence of $T_{\text {eff }}$ (max) on $Y$ has as shallow a slope as we obtained, then $Y$ is correspondingly more poorly determined by an observational determination of $T_{\text {eff }}$ ( $\max$ ). In general our results imply a somewhat higher value of $Y$ (if $Y \geqslant 0.15$ ) for a given $T_{\text {efr }}$ (max); in any case this work may give some idea of the range of uncertainty. 


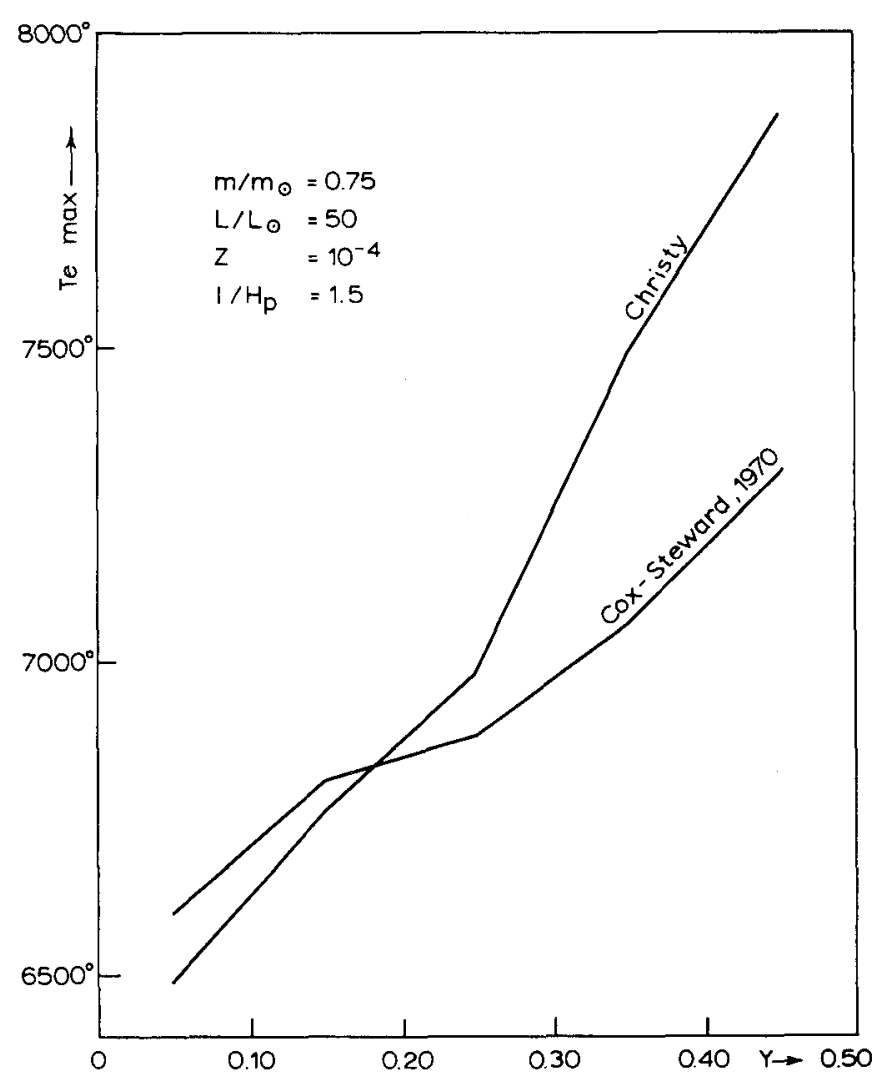

Fig. 3.

Icho Iben, Jr.: I used Christy's parameterization of old Cox-Stewart opacities in obtaining my results. It is possible that some of the differences between these results and Christy's are related to differences in the choice of boundary conditions. It may also be possible that radiation pressure was not included in the calculations which led to Christy's published results. I have used the analytic approximation to opacity for the following reasons: If one attempts to interpolate directly in Cox-Stewart tables, obtaining the opacity at any given density and temperature using only those four published points that bound the desired location, the resultant opacities are choppy and irregular. Derivatives of opacity with respect to temperature and pressure are even more choppy and irregular. They are in fact characterized by many discontinuities, with spurious changes in sign occurring frequently. Since the determination of pulsation characteristics is extremely sensitive to opacity derivatives, one simply cannot believe results gotten by interpolating in the simplest manner within the coarse meshes that are normally published. Much more sophisticated interpolation schemes must be employed. Even when this is attempted, of course, there is no guarantee that the resultant calculated opacities (and, more importantly, opacity derivatives) will adequately approximate an opacity that is calculated from first principles.

A. N. Cox: Since most people use our opacity tables, I feel a comment is in order. Let me point out that there have been two recent works on stellar opacities (Carson et al.; Watson) where opacities near $10^{6} \mathrm{~K}$ are perhaps systematically doubled. This may help Christy in his problem that pulsation calculations for the classical Cepheids give only half the mass expected from evolutionary theory. $\mathrm{He}$ has asked that the opacities be three or even four times higher at temperatures below $10^{6} \mathrm{~K}$. In regard to the opposite request, for reduced opacities at temperatures above $10^{6} \mathrm{~K}$ to help in the solar 
experiment and the horizontal branch problems, I see no present hope. It is comforting to see that we are rather in the middle of this controversy,

John Faulkner: Let me emphasize once more that the relevant, overall interior opacities seem to have been increasing in recent years. As far as stellar interior investigators are concerned, these changes have increased the difficulties of fitting the horizontal branch (too short), the Hyades main sequence binaries (too luminous) and the Sun (too many neutrinos). This is an embarrassingly large set of problems whose solution would certainly be eased if opacity reductions could be effected. 